\title{
Prevalence and risk factors for mast cell tumours in dogs in England
}

Stephanie JW Shoop ${ }^{1 *}$, Stephanie Marlow ${ }^{2}$, David B Church ${ }^{3}$, Kate English ${ }^{4}$, Paul D McGreevy ${ }^{5}$, Anneliese J Stell ${ }^{3}$, Peter C Thomson ${ }^{5}$, Dan G O'Neill ${ }^{2}$ and David C Brodbelt ${ }^{2}$

\begin{abstract}
Background: Mast cell tumour (MCT) appears to be a frequent tumour type in dogs, though there is little published in relation to its frequency in dogs in the UK. The current study aimed to investigate prevalence and risk factors for MCTs in dogs attending English primary-care veterinary practices.

Methods: Electronic patient records from practices participating in the VetCompass animal surveillance project between July 2007 and June 2013 were searched for MCT diagnosis. Various search terms and standard diagnostic terms (VeNom codes) identified records containing MCT diagnoses, which were evaluated against clinical criteria for inclusion to the study. MCT prevalence for the entire dataset and specific breed types were calculated. Descriptive statistics characterised MCT cases and multivariable logistic regression methods evaluated risk factors for association with MCT $(P<0.05)$.

Results: Within a population of 168,636 dogs, 453 had MCT, yielding a prevalence of $0.27 \%$ (95\% confidence interval (Cl) $0.24 \%-0.29 \%)$. The highest breed type specific prevalences were for the Boxer at $1.95 \%(95 \% \mathrm{Cl}$ $1.40 \%-2.51 \%)$, Golden Retriever at 1.39\% (0.98\% - 1.81\%) and Weimaraner at 0.85\% (95\% Cl $0.17 \%$ to $1.53 \%)$. Age, insurance status, neuter status, weight and breed type were associated with MCT diagnosis. Of dogs of specific breed type, the Boxer, Pug and Staffordshire Bull Terrier showed greater odds of MCT diagnosis compared with crossbred dogs. Conversely, the German Shepherd Dog, Border Collie, West Highland White Terrier, Springer Spaniel and Cocker Spaniel had reduced odds of MCT diagnosis compared with crossbred dogs. No association was found between MCT diagnosis and sex.
\end{abstract}

Clinical significance: This study highlights a clinically significant prevalence of MCT and identifies specific breed types with predisposition to MCT, potentially aiding veterinarian awareness and facilitating diagnosis.

Keywords: Mastocytoma, Mast cell, Canine, Prevalence, Primary-care practice, Epidemiology, Oncology

\section{Lay summary}

Mast cell tumour (MCT) is the most common skin tumour type in dogs, but little is known about its frequency in the general dog population nor its frequency in particular dog breeds in the UK. This study has investigated the frequency of the disease, and possible risk factors associated with the development of MCT.

This has been conducted by analysing the large number of electronic patient health records contained within

\footnotetext{
* Correspondence: stephanie.shoop@postgrad.manchester.ac.uk

'Arthritis Research UK Centre for Epidemiology, Institute of Inflammation and Repair, University of Manchester, Oxford Road, Manchester M13 9PT, England Full list of author information is available at the end of the article
}

the VetCompass animal surveillance project collected between July 2007 and June 2013.

Data were available on a population of 168,636 dogs and within this $453(0.27 \%)$ had MCT. The breeds with the highest breed specific prevalences were Boxer, Golden Retriever and Weimaraner. Conversely, some breeds appeared to be protected.

Age, insurance status, neuter status, weight and breed type were associated with MCT diagnosis. No association was found between MCT diagnosis and gender.

Such information helps to increase awareness of this condition, particularly in certain breeds, to owners and veterinarians.

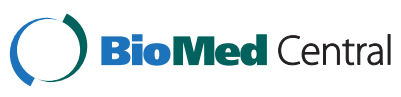

(c) 2015 Shoop et al.; licensee BioMed Central. This is an Open Access article distributed under the terms of the Creative Commons Attribution License (http://creativecommons.org/licenses/by/4.0), which permits unrestricted use, distribution, and reproduction in any medium, provided the original work is properly credited. The Creative Commons Public Domain Dedication waiver (http://creativecommons.org/publicdomain/zero/1.0/) applies to the data made available in this article, unless otherwise stated. 


\section{Background}

Mast cell tumours (MCTs) are a frequent tumour type in dogs [1], accounting for $7-21 \%$ of cutaneous tumours diagnosed [1-4]. Dobson and others (2002) reported the incidence of MCT in English dogs as 129 in 100,000 dogs per year [5]; however, this study was limited to insured animals and thus may generalise poorly to the wider population of dogs in England. MCTs occur primarily in the dermis and subcutaneous tissues and can be confirmed in $92-96 \%$ of cases through fine needle aspirate (FNA) cytology [6]. Histopathology allows description of the degree of MCT malignancy and clinical grading [6] using two grading systems. Using the Patnaik system, MCTs are categorised into three grades (I, II \& III), the third being more clinically important because tumours of this grade are often more aggressive and may metastasise [7]. The more recently proposed Kiupel two tier system categorises MCTs into high or low-grade in order to increase concordance among veterinary pathologists [8].

There are likely to be many factors contributing to the development of MCT which may be genetically influenced [6]. Up to $40 \%$ of dogs with subcutaneous and cutaneous MCTs have been found to have mutations in a proto-oncogene, $c-k i t$ [6]. Other potential factors include neuter status, sex, age, weight, insurance status and specific breed types. Females [9], and particularly neutered females [10] have been associated with increased risk of MCT in some studies, although other studies failed to identify an association between sex and MCT [11,12]. While the mean age at presentation of cutaneous MCTs has been reported at between 7.5 and 9 years old [9], one study found poorly differentiated (grade III) tumours more common in younger dogs, although the study population consisted of only one breed, the Shar-Pei [13]. Likewise, while one study found no difference in pre-disposition to MCTs between dogs of specific breed types and mixed breed (crossbred) dogs overall [14], certain breed types have previously been associated with this condition. Most commonly, Boxers and Golden Retrievers $[10,15,16]$ and other larger breed types have been associated with pre-disposition to MCT whereas smaller breed types are reported to be at lower risk of the condition [10]. Less commonly, breed types including Pugs [10,17], Weimaraners [15,16] and the mastiff and terrier phylogenetic clusters [10] have also been associated with having MCTs.

This study aimed to estimate the prevalence and explore risk-factors for the development of MCTs in dogs, using a large sample of animals attending primary-care veterinary practices in England. By identifying factors associated with a higher risk of developing MCTs, it was hoped to improve the index of suspicion for this disease and hence early recognition of this important condition.

\section{Results}

The VetCompass system documented 168,636 dogs from a total of 94 veterinary practices within England between $28^{\text {th }}$ June 2007 and $30^{\text {th }}$ June 2013. Of these, 453 dogs met the clinical criteria for inclusion to the study and were classified as dogs presenting with MCT at a participating veterinary practice during the study period. Thus, the MCT prevalence was estimated at $0.27 \%$ ( $95 \%$ CI $0.24 \%$ to $0.29 \%$ ) over the study period. Individual breed type MCT prevalences that exceeded this overall estimate included that of the Boxer at 1.95\% (95\% CI $1.40 \%$ to $2.41 \%$ ), the Golden Retriever at $1.39 \%$ (95\% CI $0.98 \%$ to $1.81 \%$ ), the Weimaraner at $0.85 \%$ ( $95 \%$ CI $0.17 \%$ to $1.53 \%$ ), the Labrador Retriever at $0.72 \%$ (95\% CI $0.58 \%$ to $0.85 \%$ ), the Staffordshire Bull Terrier at $0.51 \%$ (95\% CI $0.39 \%$ to $0.62 \%$ ) and the Pug at $0.50 \%$ (95\% CI $0.13 \%$ to $0.88 \%)$. Individual breedtype MCT prevalences that fell below the overall estimate included the Springer Spaniel at 0.20\% (95\% CI $0.06 \%$ to $0.35 \%$ ), the Jack Russell Terrier at $0.16 \%$ (95\% CI $0.09 \%$ to $0.23 \%$ ), the West Highland White Terrier at $0.07 \%$ ( $95 \% \mathrm{CI} 0.00 \%$ to $0.15 \%$ ), the Border Collie at $0.07 \%$ ( $95 \%$ CI $0.00 \%$ to $0.14 \%$ ), the Cocker Spaniel at $0.06 \%$ ( $95 \%$ CI $0.00 \%$ to $0.12 \%$ ), the Yorkshire Terrier at $0.04 \%$ (95\% CI $0.00 \%$ to $0.09 \%$ ) and the German Shepherd Dog at $0.02 \%$ (95\% CI $0.00 \%$ to 0.05 ) (Table 1A). Overall MCT prevalence for dogs of specific breed types was $0.29 \%$ (95\% CI $0.26-0.32 \%)$ and overall crossbred prevalence was $0.18 \%(95 \%$ CI $0.14-0.22)$ (Table 1B).

Of the cases, $48 \%$ were male ( $\mathrm{n}=218$ ), $64 \%$ insured $(\mathrm{n}=291)$ and $71 \%$ neutered $(\mathrm{n}=323)$. The median age at diagnosis was 8.2 years (interquartile range (IQR) 6.0 to $10.2 \mathrm{yr}$ ) and median weight $27.7 \mathrm{~kg}$ (IQR 20.0 to $34.2 \mathrm{~kg}$ ). The most common breed types affected were the Labrador Retriever $(23 \%, \mathrm{n}=106)$, Staffordshire Bull Terrier $(16 \%, \mathrm{n}=72)$, Boxer $(10 \%, \mathrm{n}=47)$, Golden Retriever (9\%, $\mathrm{n}=43)$, Jack Russell Terrier (4\%, $\mathrm{n}=18$ ), Springer Spaniel $(2 \%, \mathrm{n}=8)$, Pug $(2 \%, \mathrm{n}=7)$ and Weimaraner $(1 \%, \mathrm{n}=6)$. Fourteen percent were crossbred $(\mathrm{n}=65)$. Of the controls, $52 \%$ were male $(n=1,067), 26 \%$ insured $(\mathrm{n}=527)$ and $40 \%$ neutered $(\mathrm{n}=820)$. The median age was 3.1 years (IQR 0.5 to 7.2 yrs) and median weight $11.6 \mathrm{~kg}$ (IQR 6.0 to $24.7 \mathrm{~kg}$ ). The most common control breed types were the Labrador Retriever (11\%, $\mathrm{n}=215)$, Staffordshire Bull Terrier $(8 \%, \mathrm{n}=159)$, Jack Russell Terrier $(8 \%, \mathrm{n}=155)$, Cocker Spaniel $(4 \%, \mathrm{n}=86)$, West Highland White Terrier $(3 \%, \mathrm{n}=66)$, German Shepherd Dog $(3 \%, \mathrm{n}=63)$, Border Collie $(3 \%, \mathrm{n}=60)$, Yorkshire Terrier $(3 \%, \mathrm{n}=57)$, Springer Spaniel $(2 \%, \mathrm{n}$ $=50)$ and Golden Retriever $(2 \%, \mathrm{n}=39)$. Nineteen percent were crossbred $(\mathrm{n}=391)$. The 453 cases and 2,036 controls ( $1: 4$ case to control ratio) were taken forward for evaluation of risk factors for a diagnosis of MCT. 
Table 1 Breed-type specific prevalence of mast cell tumour (MCT) diagnosis with $95 \%$ confidence intervals (CI)

\begin{tabular}{|c|c|c|c|c|}
\hline \multicolumn{5}{|c|}{ A) Specific breed types } \\
\hline Breed-type & Cases (n) & Total (n) & MCT prevalence (\%) & $95 \% \mathrm{Cl}(\%)$ \\
\hline Boxer & 47 & 2406 & 1.95 & $1.40-2.51$ \\
\hline Golden Retriever & 43 & 3086 & 1.39 & $0.98-1.81$ \\
\hline Weimaraner & 6 & 705 & 0.85 & $0.17-1.53$ \\
\hline Labrador Retriever & 106 & 14781 & 0.72 & $0.58-0.85$ \\
\hline Staffordshire Bull Terrier & 72 & 14219 & 0.51 & $0.39-0.62$ \\
\hline Pug & 7 & 1391 & 0.50 & $0.13-0.88$ \\
\hline Springer Spaniel & 8 & 3906 & 0.20 & $0.06-0.35$ \\
\hline Jack Russell Terrier & 18 & 11333 & 0.16 & $0.09-0.23$ \\
\hline West Highland White Terrier & 3 & 4254 & 0.07 & $0.00-0.15$ \\
\hline Border Collie & 3 & 4501 & 0.07 & $0.00-0.14$ \\
\hline Cocker Spaniel & 4 & 6353 & 0.06 & $0.00-0.12$ \\
\hline Yorkshire Terrier & 2 & 5512 & 0.04 & $0.00-0.09$ \\
\hline German Shepherd Dog & 1 & 5993 & 0.02 & $0.00-0.05$ \\
\hline \multicolumn{5}{|c|}{ B) Summary results } \\
\hline Specific breed type & 388 & 132139 & 0.29 & $0.26-0.32$ \\
\hline Crossbred & 65 & 36361 & 0.18 & $0.14-0.22$ \\
\hline Unknown breed & 0 & 136 & - & - \\
\hline Total & 453 & 168636 & 0.27 & $0.24-0.29$ \\
\hline
\end{tabular}

Dogs were recruited from a VetCompass population of 168,636 dogs. Breed types are listed from highest to lowest prevalence.

\section{Risk factor analysis}

Univariable analysis identified insured and entire dogs as having significantly increased odds of MCT. Weight and age were also associated with MCT, with the categories at highest odds being 30.01 to $40.00 \mathrm{~kg}$, compared to $\leq 10.00 \mathrm{~kg}$ and 8 to 10 years of age, compared to $\leq 2.00$ years, respectively (Table 2 ). In addition, the proportion of certain breed types in case and control groups differed (Figure 1), suggesting at least a univariate association between specific breed type and MCT diagnosis. In particular, the Labrador Retriever, Staffordshire Bull Terrier, Golden Retriever, Boxer, Pug and Weimaraner appeared to be more frequently represented among cases than controls. There was also a significantly larger proportion of specific breed types compared to crossbreds in case compared to control samples. There was no significant difference in sex between the two groups (Table 2).

All variables tested showed at least a general trend ( $\mathrm{P} \leq 0.25$ ) of association with the outcome (Table 2 ), and were taken forward for evaluation in the multivariable analysis [18] (Table 3). The multivariable model identified age, insurance status, weight, neuter status and specific breeds as statistically significantly associated with MCT diagnosis. Sex was not significantly associated with MCT diagnosis $(\mathrm{P}=0.18)$.

Dogs over 10 years old had 41.3 times the odds of MCT diagnosis compared with dogs under 2 years old
(95\% CI 16.7 to 102.5 ). Dogs of weight 20 to $30 \mathrm{~kg}$ had 2.6 times the odds of MCT diagnosis compared with those under $10 \mathrm{~kg}$ (95\% CI 1.4 to 4.8 ). Insured dogs were at 3.1 times the odds (95\% CI 2.2 to 4.5 ) and neutered dogs were at 0.1 times the odds of having MCT (95\% CI 0.1 to 0.2 ) compared to uninsured and entire dogs respectively (Figure 2). Of specific breed types, the Boxer was at 10.7 times (95\% CI 3.7 to 30.4), Pug at 10.0 times (95\% CI 1.5 to 64.5) and Staffordshire Bull Terrier at 4.2 times the odds (95\% CI 2.2 to 8.2) of having MCT compared with crossbred dogs. Conversely, the German Shepherd Dog was at 0.0 times (95\% CI 0.0 to 0.4 ), Border Collie at 0.1 times (95\% CI 0.0 to 0.5 ), West Highland White Terrier at 0.2 times (95\% CI 0.0 to 0.8 ), Springer Spaniel at 0.3 times (95\% CI 0.1 to 0.9 ), and Cocker Spaniel at 0.3 times the odds (95\% CI 0.1 to 0.9 ) of having MCT compared with crossbred dogs. No association was found between sex and MCT diagnosis. Dogs of all other specific breed types other than those individually analysed were at 0.4 times the odds of having MCT than crossbreds (95\% CI 0.3-0.0.7). When comparing all specific breed types versus all crossbreds without testing for individual breed effects in the multivariable model, no significant difference in MCT predisposition was observed ( $\mathrm{P}=0.762$ ). A significant interaction was observed between weight and the specific breed type variable, but a likelihood ratio test suggested no significant 
Table 2 Risk factors for mast cell tumour (MCT) from univariable analysis

\begin{tabular}{|c|c|c|c|c|c|c|}
\hline Variable & Category & Case & Control & OR & $95 \% \mathrm{Cl}$ & P-value \\
\hline \multirow[t]{6}{*}{$\overline{\text { Age }}$} & $\leq 2.0$ years & 8 & 840 & 1 (base) & & $<0.001$ \\
\hline & 2.0-4.0 years & 22 & 301 & 7.7 & $3.4-17.4$ & $<0.001$ \\
\hline & 4.0-6.0 years & 60 & 245 & 25.7 & $12.1-54.5$ & 0.038 \\
\hline & $6.0-8.0$ years & 82 & 199 & 43.3 & $20 .-90.9$ & $<0.001$ \\
\hline & 8.0-10.0 years & 95 & 171 & 58.3 & $27.8-122.3$ & $<0.001$ \\
\hline & $>10.0$ years & 108 & 267 & 42.5 & $20.4-88.2$ & $<0.001$ \\
\hline Age (continuous) & Median (IQR) (years) & $8.2(6.0-10.2)$ & $3.1(0.5-7.2$ & 1.2 & $1.2-1.3$ & $<0.001$ \\
\hline \multirow[t]{15}{*}{ Breed Type } & Crossbred & 65 & 391 & 1 (base) & & 0.016 \\
\hline & Boxer & 47 & 22 & 12.9 & $7.3-22.7$ & $<0.001$ \\
\hline & Golden Retriever & 43 & 39 & 6.6 & $4.0-11.0$ & $<0.001$ \\
\hline & Weimaraner & 6 & 9 & 4.0 & $1.4-11.6$ & 0.028 \\
\hline & Labrador Retriever & 106 & 215 & 3.0 & $2.1-4.2$ & $<0.001$ \\
\hline & Pug & 7 & 15 & 2.8 & $1.1-7.2$ & 0.096 \\
\hline & Staffordshire Bull Terrier & 72 & 159 & 2.7 & $1.9-4.0$ & $<0.001$ \\
\hline & Springer Spaniel & 8 & 50 & 1.0 & $0.4-2.1$ & 0.379 \\
\hline & Jack Russell Terrier & 18 & 155 & 0.7 & $0.4-1.2$ & 0.006 \\
\hline & Border Collie & 3 & 60 & 0.3 & $0.1-1.0$ & 0.005 \\
\hline & Cocker Spaniel & 4 & 86 & 0.3 & $0.1-0.8$ & 0.001 \\
\hline & West Highland White Terrier & 3 & 66 & 0.3 & $0.1-0.9$ & 0.002 \\
\hline & Yorkshire Terrier & 2 & 57 & 0.2 & $0.1-0.9$ & 0.003 \\
\hline & German Shepherd Dog & 1 & 63 & 0.1 & $0.0-0.7$ & $<0.001$ \\
\hline & Other specific breed type & 68 & 649 & 0.6 & $0.4-0.9$ & 0.456 \\
\hline \multirow[t]{2}{*}{ Purebred status } & Crossbred & 65 & 391 & 1 (base) & & \\
\hline & Of breed type & 388 & 1654 & 1.4 & $1.1-1.9$ & 0.016 \\
\hline \multirow[t]{2}{*}{ Insurance status } & Not insured & 162 & 1502 & 1 (base) & & \\
\hline & Insured & 291 & 527 & 5.1 & $4.1-6.4$ & $<0.001$ \\
\hline \multirow[t]{2}{*}{ Neuter status } & Entire & 130 & 102 & 1 (base) & & \\
\hline & Neutered & 323 & 820 & 0.3 & $0.2-0.4$ & $<0.001$ \\
\hline \multirow[t]{2}{*}{ Sex } & Female & 233 & 956 & 1 (base) & & \\
\hline & Male & 218 & 1067 & 1.2 & $1.0-1.5$ & 0.090 \\
\hline \multirow[t]{5}{*}{ Weight } & $\leq 10.0 \mathrm{~kg}$ & 49 & 771 & 1 (base) & & $<0.001$ \\
\hline & $10.0-20.0 \mathrm{~kg}$ & 60 & 379 & 2.5 & $1.7-3.7$ & $<0.001$ \\
\hline & $20.0-30.0 \mathrm{~kg}$ & 145 & 296 & 7.7 & $5.4-10.9$ & $<0.001$ \\
\hline & $30.0-40.0 \mathrm{~kg}$ & 135 & 197 & 10.8 & $7.5-15.5$ & $<0.001$ \\
\hline & $>40.0 \mathrm{~kg}$ & 46 & 79 & 9.2 & $5.8-14.6$ & $<0.001$ \\
\hline Weight (continuous) (kg) & Median (IQR) & $27.7(20.0-34.2)$ & $11.6(6.0-24.7)$ & 1.1 & $1.1-1.1$ & $<0.001$ \\
\hline
\end{tabular}

Odds ratios (OR) with $95 \%$ confidence intervals $(\mathrm{Cl})$ are reported. Cases $(n=453)$ and controls $(n=2036)$ were selected from a VetCompass population of 168,636 dogs.

improvement to model fit ( $\mathrm{P}>0.5$ ), so this interaction was not retained. No other interactions were retained in the final model. Good final model fit was suggested by a Hosmer-Lemeshow test $(\mathrm{P}=0.831)$. Clustering at the clinic level was evaluated with clinic ID entered as a random effect but was not statistically significant and therefore was not retained $(\mathrm{P}=0.49)$.

\section{Discussion}

This study identified a prevalence of MCT of $0.27 \%$ ( $95 \%$ CI $0.24 \%$ to $0.29 \%$ ) over the study period and demonstrated associations between age, insurance status and specific breed types with MCT diagnosis. Highest MCT breed type prevalences were identified for the Boxer at 1.95\% (95\% CI 1.40\% - 2.51\%), Golden Retriever 


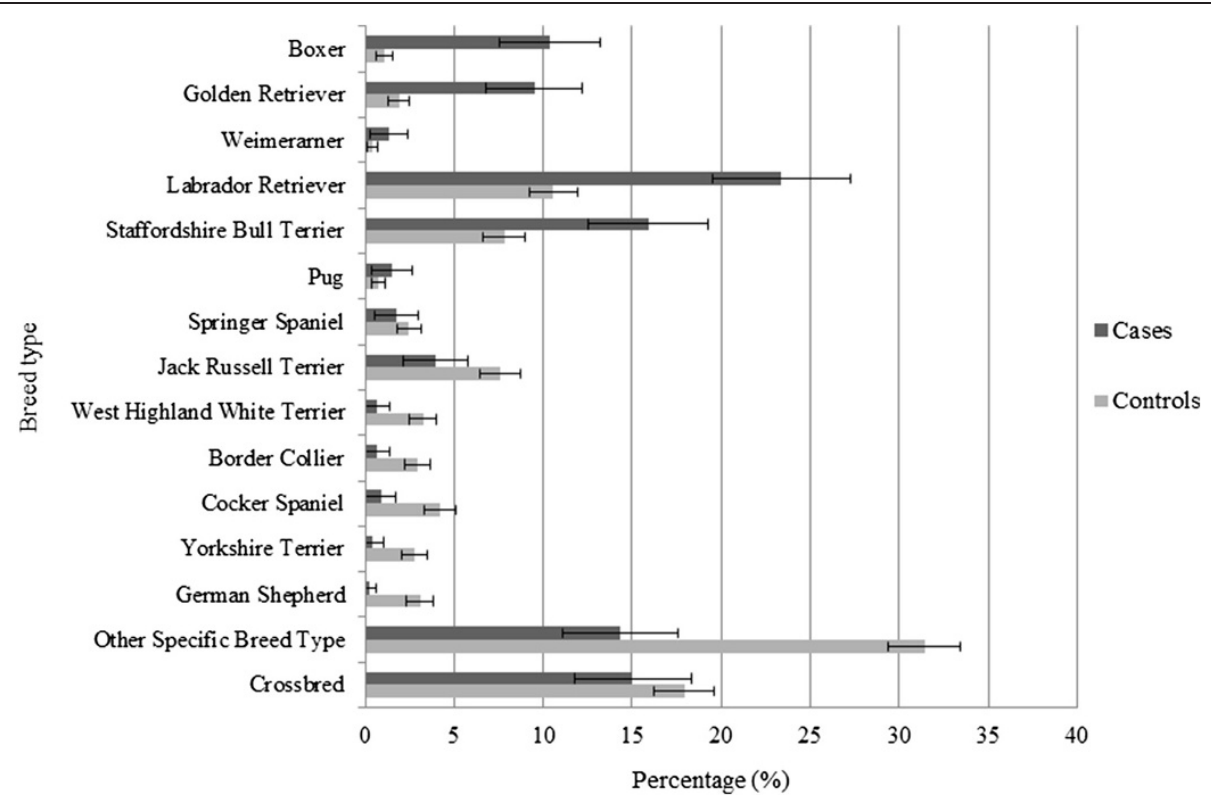

Figure 1 Percentage of mast cell tumour (MCT) positive and non-MCT dogs attributed to specific breeds. 95\% confidence intervals are displayed relating to these values. Cases $(n=453)$ and controls $(n=2036)$ were selected from a VetCompass population of 168,636 dogs.

at $1.39 \%(0.98 \%-1.81 \%)$ and Weimaraner at $0.85 \%(95 \%$ CI $0.17 \%$ to $1.53 \%)$. A previous study estimated an incidence of MCT among insured UK dogs of 129 in 100,000 dogs per year (95\% CI 107 to 145) [5]. However, as this study's population was limited to insured animals, the results may not generalise well to the overall dog population. Insured dogs are more likely to be younger [5], as policies are often cancelled in older dogs [19] and more likely to be of specific breed-types [20]. Insured dogs are also more likely to undergo further diagnostic testing and treatments [21] compared with uninsured dogs, which potentially led to an overestimation of MCT incidence by Dobson and others [5]. Conversely, insurance data may underestimate true incidence of MCT as only cases that are not excluded and whereby the deductible excess does not exceed the cost of diagnosis and treatment are included [21]. Therefore, the estimate reported in the current study is more generalisable to practice-attending dogs and to the general canine population in England. A Dutch study estimated incidence at 265 in 100,000 dog-years and focused purely on Labrador Retrievers [22]. It is likely that the latter's incidence estimation was much larger due to the potential predisposition of Labrador Retrievers to purely cutaneous MCTs [10,23,24]. Whilst Labrador Retrievers were not found to have a significant association with MCT diagnosis in the current study, it may be that this breed type is associated with only cutaneous MCTs or the current finding may highlight differences between study populations. Incidence was not calculated in the current study as it was not always clear if the cancer cases were newly diagnosed, hence it was considered more reliable to report the frequency of all existing cases in relation to all practice attending dogs during the study period and thus period prevalence. In a large study in the US, Villamil and others (2011) reported $0.25 \%$ of $1,139,616$ dogs analysed were diagnosed with cutaneous MCT [23], similar to the current study's estimation.

The current study identified that older dogs were at increased odds of MCT, with those over 10 years being around 41 times greater odds than those under 2 year of age. Previous studies have also found older dogs to be more susceptible to MCTs [23,25], consistent with the findings of the current study, with the average age for MCT diagnosis in dogs between 7.5-9 years of age [9]. In the current study, the median age of cases at presentation was 8.2 years (IQR 6.0-10.2 yr), compared to that of controls which was 3.1 years (IQR 0.5-7.2 yr). In summary, the current findings suggest that MCTs generally present in older dogs. A study focusing on Shar-Peis identified younger animals (median age 5 years) to be commonly afflicted with high grade MCTs, with $28 \%$ of cases under 2 years of age [13]. However, the small sample size $(\mathrm{n}=18)$ and single breed make it difficult to generalise the results from this earlier study.

Insured dogs had a higher odds of MCT compared with the control group in the current analysis. Biological differences between insured and uninsured dogs may have partially accounted for this association, as a higher percentage of dogs of some specific breed types have been reported to be insured compared with crossbreds [21]. Conversely, insurance cover is frequently cancelled 
Table 3 Risk factors for mast cell tumour (MCT) from final multivariable binary logistic regression model

\begin{tabular}{|c|c|c|c|c|c|c|}
\hline Variable & Category & Case & Control & OR & $95 \% \mathrm{Cl}$ & $P$ value \\
\hline \multirow[t]{6}{*}{$\overline{\text { Age }}$} & $\leq 2.0$ years & 8 & 840 & 1 (base) & & $<0.001$ \\
\hline & $2.0-4.0$ years & 22 & 301 & 5.7 & $2.1-15.2$ & 0.001 \\
\hline & $4.0-6.0$ years & 60 & 245 & 15.9 & $6.2-40.4$ & $<0.001$ \\
\hline & $6.0-8.0$ years & 82 & 199 & 18.3 & $7.3-45.9$ & $<0.001$ \\
\hline & 8.0-10.0 years & 95 & 171 & 38.3 & $15.1-97.4$ & $<0.001$ \\
\hline & $>10.0$ years & 108 & 267 & 41.3 & $16.7-102.5$ & $<0.001$ \\
\hline \multirow[t]{15}{*}{ Breed type } & Crossbred & 65 & 391 & 1 (base) & & $<0.001$ \\
\hline & Boxer & 47 & 22 & 10.7 & $3.7-30.4$ & $<0.001$ \\
\hline & Pug & 7 & 15 & 10.0 & $1.5-64.5$ & 0.016 \\
\hline & Staffordshire Bull terrier & 72 & 159 & 4.2 & $2.2-8.2$ & $<0.001$ \\
\hline & Golden Retriever & 43 & 39 & 2.0 & $0.9-4.5$ & 0.080 \\
\hline & Weimaraner & 6 & 9 & 1.4 & $0.2-9.8$ & 0.718 \\
\hline & Labrador Retriever & 106 & 215 & 1.3 & $0.8-2.3$ & 0.319 \\
\hline & Jack Russell Terrier & 18 & 155 & 0.9 & $0.4-2.1$ & 0.790 \\
\hline & Cocker Spaniel & 4 & 86 & 0.3 & $0.1-0.9$ & 0.037 \\
\hline & Springer Spaniel & 8 & 50 & 0.3 & $0.1-0.9$ & 0.029 \\
\hline & West Highland White Terrier & 3 & 66 & 0.2 & $0.0-0.8$ & 0.030 \\
\hline & Border Collie & 3 & 60 & 0.1 & $0.0-0.5$ & 0.004 \\
\hline & Yorkshire Terrier & 2 & 57 & 0.1 & $0.0-1.0$ & 0.053 \\
\hline & German Shepherd Dog & 1 & 63 & 0.0 & $0.0-0.4$ & 0.007 \\
\hline & Other specific breed type & 68 & 649 & 0.4 & $0.3-0.7$ & 0.001 \\
\hline \multirow[t]{2}{*}{ Insurance Status } & Not Insured & 162 & 1502 & 1 (base) & & \\
\hline & Insured & 291 & 527 & 3.1 & $2.2-4.5$ & $<0.001$ \\
\hline \multirow[t]{2}{*}{ Neuter Status } & Entire & 130 & 102 & 1 (base) & & \\
\hline & Neutered & 323 & 820 & 0.1 & $0.1-0.2$ & $<0.001$ \\
\hline \multirow[t]{5}{*}{ Weight } & $\leq 10.0 \mathrm{~kg}$ & 49 & 771 & 1 (base) & & 0.004 \\
\hline & $10.0-20.0 \mathrm{~kg}$ & 60 & 379 & 1.1 & $0.6-2.1$ & 0.744 \\
\hline & $20.0-30.0 \mathrm{~kg}$ & 145 & 296 & 2.6 & $1.4-4.8$ & 0.002 \\
\hline & $30.0-40.0 \mathrm{~kg}$ & 135 & 197 & 2.5 & $1.3-4.9$ & 0.007 \\
\hline & $>40.0 \mathrm{~kg}$ & 46 & 79 & 2.6 & $1.2-5.6$ & 0.017 \\
\hline
\end{tabular}

Odds ratios (OR) with 95\% confidence intervals $(\mathrm{Cl})$ are reported. Cases $(\mathrm{n}=453)$ and controls $(\mathrm{n}=2036)$ were selected from a VetCompass population of 168,636 dogs.

in older dogs and so the insured group may have underrepresented older dogs that had increased odds of MCT. However, diagnostic testing and treatments likely differed between insured and non-insured dogs. Insured dogs may have been more likely to undergo further tests that confirm MCT [21], and therefore to have met clinical criteria for inclusion to the study.

Similar to Peters and others [11] and Miller [13], the current study found no association between sex and MCT. Consistent with the findings of White and colleagues [10] and Zink and others [26], the current study also reported an association between neuter status and MCT development. However, whilst Zink and others [26] reported increased odds of MCT for neutered
Vizslas and White and others [10] reported an increased risk of MCT for neutered females, the current study found decreased odds of MCT for neutered dogs in general. However, limitations of the present study may also contribute to these conflicting findings. In the practice management systems, each dog was assigned one neuter status which was promptly updated without notification of date when this status changed. At the time of the data query, neuter status may have been different from that when initial MCT diagnosis was made, therefore this study may have underestimated the number of dogs with MCT that were entire at diagnosis. Further investigation into a potential association between neuter status in both sexes and MCT diagnosis is merited. 


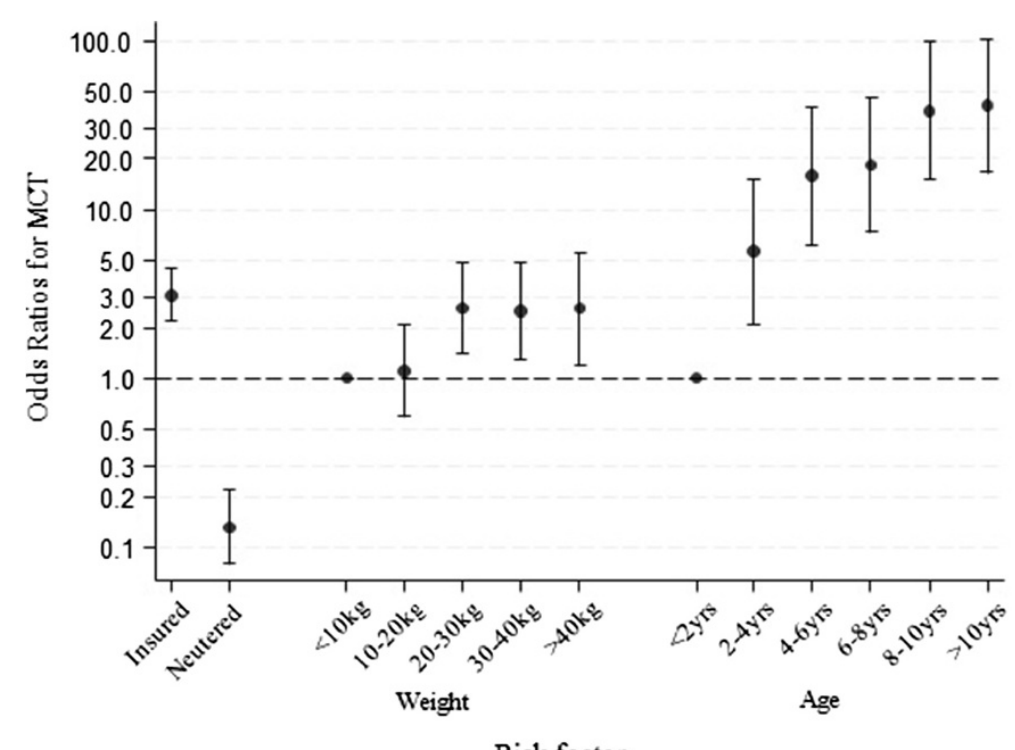

Risk factor

Figure 2 Risk factors for mast cell tumour (MCT) from final multivariable binary logistic regression model. Odds ratios for MCT with corresponding 95\% confidence intervals are reported. Dogs weighing less than $10 \mathrm{~kg}$ and aged less than 2 yrs have odds ratios of 1 as were used as baseline categories for weight and age variables respectively.

Previous studies describing risk-factors for MCT were mainly limited to specific breed types, focused purely on cutaneous MCTs and had smaller sample sizes than the current study. Larger-sized breeds were previously reported at higher risk of developing MCT than smaller and medium breeds [10]. Similarly, the current study found that dogs over $20 \mathrm{~kg}$ had over twice the odds of having MCT compared to those under $10 \mathrm{~kg}$. The current study also supports the findings of a large US study of no difference in MCT diagnosis between dogs of specific breed types and crossbred dogs in general [14]. This is likely a product of increased risk for a few specific breed types and decreased risk for the majority of dogs of other breed types, supported by the significantly decreased MCT odds for the 'other specific breed type' category in the present analysis.

The current study supports the general consensus that Boxers have increased risk of MCTs [10,11,15,16,23,25,27]. Consistent with previous work [11,16,23], the study also identified Staffordshire Bull Terriers as being at higher odds and it is hypothesised that their predisposition, including that of Boxers and other bull-type breeds, may be linked to common ancestry [11] since these breed types are closely phylogenetically clustered [27]. As also reported by Murphy and others [16], Villamil and others [23], White and others [10] and McNiel and others [17], the current study identified Pugs as having high odds of developing MCTs. Golden Retrievers [10,15,16,23,27], Labrador Retrievers $[10,23,24]$ and Weimaraners $[15,16,27]$ have previously been found to be at high risk of cutaneous MCTs. Though the current study reports trends supporting these findings, it did not detect a statistically significant association for the Golden Retriever, Labrador Retriever or the Weimaraner compared to crossbred dogs. However, given the small sample size for Weimaraners (case $\mathrm{n}=6$, control $\mathrm{n}=9$ ), the current study may have had limited power to explore infrequently presented breed types and smaller magnitudes of association. This study corroborates previous work that found German Shepherd Dogs [23,27], Cocker Spaniels, West Highland White Terriers and Border Collies to be at decreased odds of MCT [27] compared to crossbred dogs. However, in contrast to previous work this study did not detect a reduction in odds of MCT in Yorkshire Terriers [10,23].

Some limitations to the current study are worth noting. When assessing common breed types in control samples, a number had small sample sizes in the case group. Border Collies, German Shepherd Dogs, Weimaraners, West Highland White Terriers and Yorkshire Terriers were analysed separately because previous studies suggested protective effects for these breed types [10,23,27]. This may be an effect of partial confounding by age. The case group will have had older dogs than the control group as older dogs were at greater odds of MCT and therefore fewer dogs of large breed type, which have been reported to have shorter longevity [28]. However, age of dogs was retained in the final multivariable model and should have, at least in part, adjusted for this association between breed and age structure. Matching cases to controls based on a similar age-structure may have improved interpretation of breed type associations with MCT, but would have prevented investigation into any association between age and 
this condition. Based on the stated case definition, the prevalence of MCT was likely to be under-estimated. Subcutaneous tumours may be less likely to be diagnosed compared with cutaneous MCTs because of their less prominent nature. In addition, some dogs with MCT will have been excluded from the case sample where a diagnosis of MCT was made but no definitive laboratory results from FNA or histopathology were recorded. This included dogs where a history of MCT only was cited and where a MCT diagnosis was recorded but euthanasia followed without a definitive test. Likewise, in a small number of dogs, an impression smear alone was used to diagnose MCT. Whilst in theory an MCT diagnosis could be obtained from an ulcerated mass through an impression smear, if there is superficial inflammation associated with the lesion, the smear may be difficult to interpret [29]. Conversely, it was possible that controls may have had MCTs, but by excluding dogs with any mention of mast cell in their case notes, this study aimed to minimise the number of misclassified controls. Insurance status of study dogs may not have been completely accurate. Where there were no insurance data at the date of diagnosis for case dogs, the nearest possible previous insurance status was used. Thus, insurance status may have changed without documentation by the date of diagnosis. For controls, the nearest possible insurance status was used as the date of first presentation to a veterinarian.

\section{Conclusion}

This study estimated a clinically relevant prevalence of MCT in dogs attending primary-care practices in England at approximately $0.27 \%$ (95\% CI $0.24 \%$ to $0.29 \%$ ) and identified associations with older, insured, heavier dogs, and specific breed types with diagnosis. Boxers, Pugs and Staffordshire Bull Terriers were at increased odds of MCT compared to crossbred dogs. Conversely, German Shepherd Dogs, West Highland White Terriers, Border Collies and Cocker Spaniels were at lower odds of MCT. Further investigation is warranted to explore MCT occurrence in less common breed types as well as associations with subcutaneous MCT and malignancy severity.

\section{Method}

The study population was selected from the VetCompass animal surveillance database [30], which documents clinical records from English primary-care veterinary practices. Data relating to clinical consultations between $28^{\text {th }}$ June 2007 and $30^{\text {th }}$ June 2013 were collected from a group of practices using VetCompass predominantly in central and south eastern England. Electronic patient record (EPR) data included a summary diagnosis term selected from a list of standardised veterinary nomenclature (VeNom) codes, developed by the VeNom Coding Group [31], which were selected by clinicians at the time of the 'episodes of care'. Additional clinical fields collected included unique animal and clinic identification numbers, date of birth, breed type, sex, neuter status, weight, insurance status, episode of care date, clinical notes and details of specific diagnosis, confirmatory testing methods and treatments including any surgical procedures. Institutional ethics approval was gained (RVC URN 2001 1101).

The case definition for MCT included evidence of a final diagnosis of MCT recorded either by a veterinarian within the clinical notes or on an insurance claim submission. Additionally, evidence of results from a combination of fine needle aspirate (FNA) cytology and/or biopsy histopathology was required to confirm this diagnosis. Dogs that had confirmatory testing using only impression smears were not included due to difficulty in the interpretation of such smears [29]. Cases were identified from the clinical notes based on this case definition, via the standard diagnosis (VeNom codes) and the clinical free text notes. Search terms applied for the free text clinical notes included 'mast cell', 'mast_,' 'mct' and 'masto'.

Control animals were selected using a random number generator [32] from dogs not diagnosed with MCT. Since minimal increase in statistical power can be gained beyond four controls per case [33], a subset of control animals were selected at a 1:4 case to control ratio [34]. Controls were not matched to cases on any clinical or phenotypical criteria in order to avoid excluding any potential risk factors for MCT from the analysis. Therefore, characteristics of controls derived randomly from the VetCompass database were likely to reflect the general practice attending canine population. These random numbers were then associated with unique patient identification numbers using the VLOOKUP function in Microsoft Excel 2010 [35]. Controls were excluded if they had evidence of suspicion or confirmation of MCT or no evidence of presentation to a veterinary surgeon within the existing case notes. Risk factors tested for association with MCT were age, sex, insurance status, neuter status, weight and breed type.

Prevalence of MCT diagnosis was estimated from the number of cases divided by the population of dogs documented in the VetCompass system throughout the study period. Prevalence of MCT diagnosis for individual breed types over the study period was calculated from the number of MCT cases of a specific breed type divided by the population of dogs of the same breed type in the VetCompass system presenting to participating practices during the study period. A nested case-control study design identified risk factors associated with MCT diagnosis.

\section{Data analysis}

Before importation into IBM SPSS version 20 statistical software for analysis [36], data were imported into Microsoft Excel 2010 to be cleaned and to produce one 
record per dog. Dates of confirmation of MCT diagnosis for cases, and for controls dates of first recorded presentation to the veterinarian were used in combination with dates of birth to calculate ages. Insurance status on dates that directly preceded the first date of confirmed diagnosis was used for cases and insurance status nearest the first presentation was used for controls. Weights used were those documented nearest the date of diagnosis confirmation for cases and first presentation for controls. Breed type was recorded at the originating practices based on consensus between the practice and owners. The breed types recorded were mapped to the specific breed terms within the VeNom coding system during the analysis and were reduced into 15 categories. These categories derived from the top 10 most common case breed types and top 10 most common control breed types along with a 'other specific breed type' and a 'crossbred' category, defined as dogs of mixed breed. 'Crossbred' dogs were retained as a separate group and used as the comparator group for the breed-type analyses because of a prevailing opinion that health characteristic may vary between dogs of specific breed types and crossbred dogs due to a higher degree of homozygosity within the breed types $[14,37,38]$. Crossbreds included all dogs that were not classified as breed types regardless of the suspected number of progenitor breeds in the parentage and were used as the comparator group based on their suspected higher general degree of heterozygosity. Further separation of crossbreds into those dogs with just two specific parental breed types and those with more mixed ancestry was not possible at this stage.

Univariable analysis assessed associations between risk factors and a diagnosis of MCT using Chi-squared and Mann-Whitney U-test for categorical and quantitative data respectively because data were non-normally distributed. Broadly significant variables from the univariable analysis $(\mathrm{P} \leq 0.25)$ were taken forward for consideration in the multivariable logistic regression model [18]. Collinearity of variables taken forward was explored via standard statistical methods [39]. A manual forward selection step-wise construction method was taken for model building with statistical significance set at the $5 \%$ level. The forward step-wise regression used the likelihood ratio test. Clustering at the clinic level was evaluated with the addition of clinic ID as a random effect. Final variables were evaluated for pairwise interactions and the final model was evaluated with the Hosmer-Lemeshow goodness-of-fit test [40].

\section{Abbreviations}

Cl: Confidence interval; EPR: Electronic patient record; MCT: Mast cell tumour; VeNom: Veterinary nomenclature.

\section{Competing interests}

The authors declare that they have no competing interests.

\section{Authors' contributions}

SJWS is the primary author for the current work and was involved in the conception, design, data collection, data analysis and drafting of the manuscript. SM contributed to the conception and design of the work, and helped to draft and revise the manuscript. DBC contributed to the conception and design of the work, and helped to draft and revise the manuscript. KE contributed to the conception and design of the work, and helped to draft and revise the manuscript. DGON Was involved in the conception, design, data collection, supervision of data analysis, drafting and revising the manuscript. PDM contributed to the conception and design of the work, and helped to draft and revise the manuscript. AS contributed to the conception and design of the work, and helped to draft and revise the manuscript. PCT contributed to the conception and design of the work, and helped to draft and revise the manuscript. DCB was involved in the conception, design, data collection, supervision of data analysis, drafting and revising the manuscript. All authors read and approved the final manuscript.

\section{Acknowledgements}

The authors would like to thank those practices and clients of the Medivet Veterinary Partnership and other participating VetCompass practices for their participation in this study. We also thank Peter Dron for database development and Noel Kennedy for assistance with VetCompass database management and programming (Royal Veterinary College).

\section{Author details}

${ }^{1}$ Arthritis Research UK Centre for Epidemiology, Institute of Inflammation and Repair, University of Manchester, Oxford Road, Manchester M13 9PT, England. ${ }^{2}$ Veterinary Epidemiology, Economics and Population Health, The Royal Veterinary College, Hawkshead Lane, North Mymms, Hatfield, Herts AL9 7TA, England. ${ }^{3}$ Small Animal Medicine and Surgery Group, The Royal Veterinary College, Hawkshead Lane, North Mymms, Hatfield, Herts AL9 7TA, England. ${ }^{4}$ Pathology and Pathogen Biology, The Royal Veterinary College, Hawkshead Lane, North Mymms, Hatfield, Herts AL9 7TA, England. ${ }^{5}$ R.M.C. Gunn Building (B19), Faculty of Veterinary Science, The University of Sydney, Sydney, NSW 2006, Australia

Received: 25 June 2014 Accepted: 18 November 2014 Published: 26 January 2015

\section{References}

1. Amagai Y, Tanaka A, Matsuda A, Oida K, Jung K, Matsuda $H$. The phosphoinositide 3-kinase pathway is crucial for the growth of canine mast cell tumors. J Vet Med Sci. 2013;75(6):791-4.

2. Cohen D, Reif JS, Brodey RS, Keiser H. Epidemiological analysis of the most prevalent sites and types of canine neoplasia observed in a veterinary hospital. Cancer Res. 1974;34(11):2859-68.

3. Bostock DE. Neoplasms of the skin and subcutaneous tissues in dogs and cats. Br Vet J. 1986;142(1):1-19.

4. Lin WW, Karin M. A cytokine-mediated link between innate immunity, inflammation, and cancer. J Clin Invest. 2007;117(5):1175-83.

5. Dobson JM, Samuel S, Milstein H, Rogers K, Wood JL. Canine neoplasia in the UK: estimates of incidence rates from a population of insured dogs. J Small Anim Pract. 2002:43(6):240-6.

6. Welle MM, Bley CR, Howard J, Rüfenacht S. Canine mast cell tumours: a review of the pathogenesis, clinical features, pathology and treatment. Vet Dermatol. 2008;19(6):321-39.

7. Withrow SJ, Vail DM, Page R. Mast cell tumours. In: Withrow and MacEwen's small animal clinical oncology. Fourth edition. Missouri: Saunders Elsevier; 2007.

8. Kuipel M, Webster JD, Bailey KL, Best S, DeLay J, Detrisac CJ, et al. Proposal of a 2-tier Histologic grading system for canine cutaneous mast cell tumors to more accurately predict biological behavior. Vet Pathol. 2011;48(1):147-55

9. O'Connell K, Thomson M. Evaluation of prognostic indicators in dogs with multiple, simultaneously occurring cutaneous mast cell tumours: 63 cases. Vet Comp Oncol. 2013;11(1):51-62.

10. White CR, Hohenhaus AE, Kelsey J, Procter-Gray E. Cutaneous MCTs: associations with spay/neuter status, breed, body size, and phylogenetic cluster. J Am Anim Hosp Assoc. 2011;47(3):210-6.

11. Peters JA. Canine mastocytoma: excess risk as related to ancestry. J Natl Cancer Inst. 1969;42(3):435-43. 
12. Patnaik AK, Ehler WJ, MacEwen EG. Canine cutaneous mast cell tumor: morphologic grading and survival time in 83 dogs. Vet Pathol. 1984;21(5):469-74.

13. Miller DM. The occurrence of mast cell tumors in young shar-peis. J Vet Diagn Invest. 1995;7(3):360-3.

14. Bellumori TP, Famula TR, Bannasch DL, Belanger JM, Oberbauer AM. Prevalence of inherited disorders among mixed-breed and purebred dogs: 27,254 cases (1995-2010). J Am Vet Med Assoc. 2013;242(11):1549-55.

15. Kiupel M, Webster JD, Miller RA, Kaneene JB. Impact of tumour depth, tumour location and multiple synchronous masses on the prognosis of canine cutaneous mast cell tumours. J Vet Med A Physiol Pathol Clin Med. 2005;52(6):280-6.

16. Murphy S, Sparkes AH, Blunden AS, Brearley MJ, Smith KC. Effects of stage and number of tumours on prognosis of dogs with cutaneous mast cell tumours. Vet Rec. 2006;158(9):287-91.

17. McNiel EA, Prink AL, O'Brien TD. Evaluation of risk and clinical outcome of mast cell tumours in pug dogs. Vet Comp Oncol. 2006;4(1):2-8.

18. Dohoo I, Martin W, Stryhn H. Logistic regression. In: Veterinary epidemiologic research. Second edition. Prince Edward Island, Canada: VER Inc; 2010.

19. Egenvall A, Bonnett BN, Hedhammar $\AA$, Olson P. Mortality in over 350,000 insured swedish dogs from 1995-2000: II. Breed-specific Age and survival patterns and relative risk for causes of death. Acta Veterinaria Scandinavica. 2005:46(3):121-36.

20. Egenvall A, Headhammer $\AA$, Bonnett BN, Olson P. Survey of the swedish dog population: age, gender, breed, location and enrollment in animal insurance. Acta Vet Scand. 1999;40(3):231-40.

21. Egenvall A, Nødtvedt A, Penell J, Gunnarsson L, Bonnett BN. Insurance data for research in companion animals: benefits and limitations. Acta Veterinaria Scandinavica. 2009:51(1):42.

22. Boerkamp KM, Teske E, Boon LR, Grinwis GCM, van den Bossche L, Rutteman GR. Estimated incidence rate and distribution of tumours in 4,653 cases of archival submissions derived from the Dutch golden retriever population. BMC Vet Res. 2014;10:34

23. Villamil JA, Henry CJ, Bryan JN, Ellersieck M, Schultz L, Tyler JW, et al. Identification of the most common cutaneous neoplasms in dogs and evaluation of breed and age distributions for selected neoplasms. J Am Vet Med Assoc. 2011:239(7):960-5.

24. Wakshlag JJ, Rassnick KM, Malone EK, Struble AM, Vachhani P, Trump DL, et al. Cross-sectional study to investigate the association between vitamin D status and cutaneous mast cell tumours in Labrador retrievers. Br J Nutr. 2011;106 Suppl 1:S60-3.

25. Misdorp W. Mast cells and canine mast cell tumours. A review Vet Q 2004;26(4):156-69.

26. Zinc MC, Farhoody P, Elser SE, Ruffini LD, Gibbons TA, Rieger RH. Evaluation of the risk and age of onset of cancer and behavioral disorders in gonadectomized vizslas. J Am Vet Med Assoc. 2014;244(3):309-19.

27. Dobson JM. Breed-predispositions to cancer in pedigree dogs. ISRN Vet Sci. 2013;2013:Article ID: 941275.

28. O'Neill DG, Church DB, McGreevy PD, Thomson PC, Brodbelt DC. Longevity and mortality of owned dogs in England. Vet J. 2013;198(3):638-43.

29. Pohlman LM. Minimizing those "nondiagnostic" cytology sampes. In: Western veterinary conference. Manhattan, USA: Kansas State University; 2013

30. Vetcompass. Vetcompass: health surveillance for UK companion animals [http://www.rvc.ac.ukNetcompass/]

31. Venom Coding Group. VeNom veterinary nomeclature [http://www.venomcoding.org]

32. Haahr M. True random number service [http://www.random.org]

33. Wacholder S, Silverman DT, McLaughlin JK, Mandel JS. Selection of controls in case-control studies. III. Design options. Am J Epidemiol. 1992;135(9):1042-50.

34. Dohoo I, Martin W, Stryhn H. Case-control studies. In: Veterinary epidemiologic research. Second edition. VER Inc: Prince Edward Island, Canada; 2010.

35. Microsoft Corp. Microsoft excel 2010. Redmond, WA: Microsoft Corp; 2013

36. IBM Corp. IBM SPSS version 20. Armonk, NY: IBM Corp; 2013.

37. Bateson P. Independent inquiry into Dog breeding. Cambridge: University of Cambridge; 2010.
38. O'Neill DG, Church DB, McGreevy PD, Thomson PC, Brodbelt DC. Prevalence of disorders recorded in dogs attending primary-care veterinary practices in England. PLoS ONE. 2014;9(3):e90501. doi:10.1371/journal.pone.0090501.

39. Field A. Logistic regression. In: Discovering statistics using SPSS. Second edition. Secondth ed. London: SAGE Publications Ltd; 2005.

40. Hosmer DW, Lemeshow S. Interpretation of the fitted logistic regression model. In: Applied Logistic Regression. Second Edition. Hoboken, NJ, USA: John Wiley \& Sons; 2000.

doi:10.1186/2052-6687-2-1

Cite this article as: Shoop et al:: Prevalence and risk factors for mast cell tumours in dogs in England. Canine Genetics and Epidemiology 2015 2:1.

\section{Submit your next manuscript to BioMed Central and take full advantage of:}

- Convenient online submission

- Thorough peer review

- No space constraints or color figure charges

- Immediate publication on acceptance

- Inclusion in PubMed, CAS, Scopus and Google Scholar

- Research which is freely available for redistribution

Submit your manuscript at www.biomedcentral.com/submit
C Biomed Central 